Opinion

\title{
Imagining Life with a Genetic Disorder: The Challenge of Evaluating Health States that Exist from Birth
}

Diane B. Paul ${ }^{1,2, *}$

1. University of Massachusetts Boston, Boston, MA, USA; E-Mail: diane.paul@umb.edu

2. Museum of Comparative Zoology, Harvard University, Cambridge, MA, USA

* Correspondence: Diane B. Paul; E-Mail: diane.paul@umb.edu

Academic Editor: Joep Geraedts

Special Issue: Policy Issues in Prenatal and Neonatal Testing, Counseling, and Decision-Making

OBM Genetics

2021, volume 5 , issue 2

doi:10.21926/obm.genet. 2102130
Received: March 29, 2021

Accepted: May 11, 2021

Published: May 14, 2021

\begin{abstract}
This article explores difficulties encountered by those with no personal experience of a chronic disease or disability in accurately evaluating the quality of life with a condition present from birth. In most countries, cost-effectiveness analysis relies on ratings of health states by members of the general population, who must try to imagine what life for those affected is like based on (usually brief) descriptions of the conditions. That task is challenging for reasons that have been well-discussed in the literature. This article employs a detailed case-study of dietary treatment for the genetic disease phenylketonuria to identify additional, unrecognized challenges that arise in the case of conditions experienced from birth (or early childhood) and considers some implications of these difficulties for practices in health-state valuation.
\end{abstract}

\section{Keywords}

Health-state valuation; cost-effectiveness analysis; QALYs; public preferences; hypothetical patients; affective forecasting, disability; adaptation; genetic disease; phenylketonuria

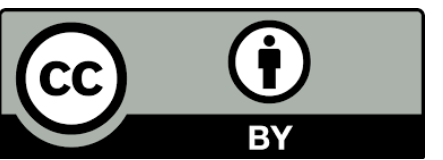

(C) 2021 by the author. This is an open access article distributed under the conditions of the Creative Commons by Attribution License, which permits unrestricted use, distribution, and reproduction in any medium or format, provided the original work is correctly cited. 


\section{Introduction}

In his classic essay "What is it like to be a bat?" philosopher Thomas Nagel argued that we cannot help extrapolating from our own experiences when we try to imagine what it is like to be another type of being and that such extrapolation is necessarily incomplete. Although we can draw certain inferences about the nature of a bat's perceptions, drives, feelings, and so forth from its physical structure and behavior, these have for the bat "a specific subjective character, which it is beyond our ability to conceive." Trying to imagine what it is like to navigate the world through sonar or to hang upside down all day by one's feet exceeds the capacity of our mental resources, for insofar as we can imagine these experiences, we can only imagine what they would be like for us, and not for the bat. And in a little-noticed aside, Nagel commented that this problem is not limited to bats (or wasps or extraterrestrial beings) but also exists between persons, such that: "The subjective character of the experience of a person deaf and blind from birth is not accessible to me, for example, nor presumably is mine to him" [1].

This article follows Nagel in arguing that to imagine what it is like to live with a condition that is experienced from birth (or additionally, acquired early in life) is exceedingly difficult and perhaps impossible, and that this claim, if true, has implications for theory and practice in any domain, including education, employment, health care, and the law, where the question arises of whether to privilege "insider" or "outsider" perspectives in evaluating specific health states. It arises with particular passion in respect to prenatal testing. In that domain, activists both for disability rights and for restrictions on access to abortion (groups that are not generally politically allied) tend to disapprove of selective abortion for fetal anomaly, and in support of that stance often cite studies [2] reporting that individuals with Down Syndrome (DS) and their families rate the quality of life [QoL] with DS higher than do medical professionals and others without experiential knowledge of the condition.

But the implications are perhaps starkest for cost-effectiveness analysis (CEA), where the need to decide whose health-state valuations should count is unavoidable. In CEA, health-state valuations (or more technically, health utility ratings) elicited from randomly chosen members of the public are generally privileged over valuations elicited from patients or their surrogates. In that regard, practice in CEA has increasingly diverged from trends elsewhere in health-care and the culture as a whole. Thus, in the law, the humanities, and the social sciences, it has become increasingly taken for granted that "outsiders" are often mistaken in their assessments and that the "insider" experiential view should thus be favored in valuing health states. Law and disability-studies scholar Elizabeth Emens expresses an increasingly common viewpoint in academia when she writes: "Those on the inside and the outside of disability often look differently at the experience, the theory, and the law of disability. From the outside, disability commonly looks like an unhappy place created by an individual medical problem for which the law sometimes provides special benefits to that individual. From the inside, disability often looks like a mundane feature of a no-less-happy life, rendered inconvenient or disabling largely by interactions with the surrounding environment, which legal accommodations alter in ways that sometimes provide benefits to many" [3]. In medicine as well, the trend over time has been to accord increasing weight to patient knowledge and preferences. However, as acknowledged by the US Panel on Cost Effectiveness in Health and Medicine (hereafter US Panel), the theory and practice of CEA represents a striking exception [4]. 
The essay begins with a brief account of the evaluative-source issue as it arises in CEA. It then summarizes the challenges encountered by those asked to value non-experienced health states (irrespective of their etiology) followed by a consideration of the additional challenges that arise in valuing health states that exist from birth or early childhood. A concrete case-the genetic disease phenylketonuria (PKU) -is used to illustrate both obstacles to creating health-state descriptions adequate for outsiders to make genuinely informed QoL assessments and the special obstacles encountered in imagining what life is like when the condition is genetic/congenital or earlyacquired. It concludes with some reflections on the implications of taking these difficulties seriously.

\section{Patient and Public Preferences in CEA}

In CEA, it is impossible to escape the issue of evaluative source. Health outcomes in this domain are typically measured in terms of the number of quality-adjusted life years (QALYs) gained. To calculate QALYS, which combine data on survival and quality of life in a single measure, it is necessary to obtain valuations of health states from some source, usually by means of a survey in which respondents are asked either how much time alive they would forgo to regain full health/function or what risk of immediate death they would accept to avoid the health state in question (known respectively as the "time trade-off" [TTO] and "standard gamble" [SG] methods). Although health professionals and caregivers are sometimes cited as groups with relevant experiences, the options on offer are typically patients on the one hand (or, in the case of children and others who lack decisional capacity, their surrogates) and a representative group of ordinary citizens on the other.

Even in CEA, the question of who should be asked to evaluate health states has always been contested [5]. However, the standard recommendation in most countries has been and still is to use what are known as "public" or "community" preferences-i.e., health-state preferences elicited from representative samples of the general population-in making resource-allocation decisions. Of course, the options are not mutually exclusive, and it is sometimes proposed that patient preferences be incorporated in special circumstances or that both patient and community preferences should be elicited. Thus, Erik Nord and colleagues suggest that the debate about whom to ask has conflated the issue of how best to measure the quality of life associated with different health states with the issue of how to measure distributive preferences in respect to resource allocation [6-8]). But in general, leading textbooks on CEA and guidelines issued by a host of official panels and agencies such as the UK National Institute for Health and Clinical Excellence (NICE), the Canadian Agency for Drugs and Technologies in Health (CADTH), the World Health Organization (WHO), and in the United States, both the Preventive Services Task Force and the US Panel continue to favor the use of community preferences over those elicited from samples of patients or their surrogates in deciding where funds are best spent. (The case for community preferences involves a mix of normative, technical, and practical considerations. This theoretical framework is summarized in the US Panel report [9]; for a critique, see [10]).

The issue of evaluative source matters in CEA because those with a chronic disease or disability tend to assign higher values to these states (i.e., view them more positively) than do those for whom the issue is only hypothetical [11-14]. The evidence is not clear-cut. Some studies find no disparity, and there are important exceptions to the general rule, including depression, chronic pain, spinal cord injury, and traumatic brain injury [12, 15-17]. Moreover, former patients, such as those with 
reversed colostomies, sometimes rate the quality of life with the condition lower than do current patients [18]. Nevertheless, the majority of empirical studies indicate that when those who have not experienced a particular health-state are asked to rate it based only on descriptions of the state and its consequences, they are willing to trade off more lifetime years or run a greater risk of immediate death to avoid it than are those with such experience. In other domains, this state of affairs, in which many people with serious, chronic disabilities state that they enjoy a good or superior quality of life "when to most external observers these individuals seem to live an undesirable daily existence," is sometimes termed the "disability paradox" [19].

The disparity in insider and outsider evaluations is usually attributed to two factors, which are weighted variously by different commentators. One factor is simply the greater knowledge of what it is like to live with a condition possessed by those with direct experience of it. Another is that most individuals eventually adapt well to chronic disease or disability and eventually feel that they experience a satisfactory quality of life.

Over time, the affected generally come to focus on previously inconceivable positives: the ways it brings family and friends closer, builds character, and enhances their appreciation of the hitherto taken-for-granted. Many will also learn new skills, participate in new activities, and develop new goals. (Adaptation may also take more problematic forms, such as cognitive denial and reduced expectations). Indeed, those affected sometimes report a higher quality of life and greater satisfaction than do the unaffected, not simply greater satisfaction than what other people think they would report. In some cases, they even state that, overall, the disease or disability has improved their lives [20]. As they learn to adjust to living with the disease or disability, their reference points may also shift such that the meaning of what it is to be in good health comes to differ from its meaning for unaffected individuals. (Although this process is often considered an aspect of adaptation, some commentators regard it as a separate factor). But these potential shifts are rarely taken into account by those without experience of the condition. Thus, a common suggestion for improving CEA is to incorporate information (or more or different kinds of information) about the process of adaptation in health-state descriptions presented to generalpopulation survey respondents and/or to provide them with information about how patients themselves rate the health states e.g. [21, 22].

\section{Hypothetical Patients: Cognitive and Affective Challenges}

In general, people are quite poor at predicting how they would feel in a future, hypothetical situation, such as getting married (or divorced), acing (or failing) a test, having one's candidate win (or lose) an election, or being hired for (or fired from) a particular job. Errors in "affective forecasting" - predicting the intensity and duration of one's emotional response to both negative and positive events-abound in many domains, including health [23,24]. Consider the oft-cited case of paraplegia. When asked to rate the life satisfaction of paraplegics, those with no experience of the condition judge paraplegics to be much unhappier than do either paraplegics themselves or those with a paraplegic friend or relative, whose assessments are based on actual observations. (However, this error of affective forecasting should not be confused with the claim that most paraplegics are as satisfied with their lives as are controls or as they were before becoming disabled [25].) One reason for the discrepancy may be that when the healthy are asked to imagine what it is like to live with paraplegia (or cancer or deafness or without a leg), they experience a shock that 
leads them to focus on the negatives. In such cases, their judgment is subject to what David Schkade and Daniel Kahneman termed the focusing illusion, in which we pay attention to one aspect of an experience, ignoring others that may have a much greater effect on well-being. Those with a chronic disease or disability, on the other hand, tend to consider the larger picture [26, 27]. (But for a skeptical perspective see Peter Ubel et al. [28]).

Nor are outsiders likely to take changes over time into account. Adaptation is a process, and those who try to imagine what life would be like if they lost their mobility, hearing, or sight (or conversely, won a lottery) are more likely to imagine how they would feel when it happened than how they would feel a year later. Thus, Daniel Gilbert and Timothy Wilson explain: "The problem with imagining only the early moments of an event is that hedonic reactions to events typically dissipate over time, which means that mental simulations tend to overrepresent the moments that evoke the most intense pleasure or pain. ... When people imagine 'losing mobility,' they expect to be less happy than people who have experienced these events actually are because their simulations overrepresent the initial-and typically the worst-moments of these events. The tendency to underestimate how quickly we will adapt to a wide range of pleasurable and painful events is probably the most commonly observed error in research on hedonic prediction" [29].

Apart from biases that produce errors of affective forecasting, trying to imagine what it is like to be in a specific impaired state is also cognitively demanding. To minimize the burden, the healthstate descriptions on which respondents rely are generally designed to be simple. Thus, the influential Global Burden of Disease (GBD) study [30], uses health-state descriptions that are meant to capture "the essential domains of loss of function in fewer than 35 words, as pilot testing indicated this was the maximum of information respondents were able to absorb" [31]. (The GBD study estimates disability adjusted life years [DALYs] rather than QALYs). Survey respondents are rarely if ever provided with information on patients' experiences of living in the health-state [22]. Nor do they have an opportunity to reflect or to learn. Daniel Hausman notes that, unlike "typical applications of consumer choice theory, in which the consumer's evaluation of commodities derives from repeated choices, with opportunities for learning and correcting errors, the preferences expressed in health measurement surveys concern alternatives about which individuals know comparatively little," and he concludes that most people lack the knowledge of particular health states (including their social, recreational, and career dimensions) that are essential for reliable valuations [32, 33].

Moreover, there is no reason to assume that such valuations are stable over time. That respondents must answer questions that they have only just encountered, with little preparation and no opportunity to reflect, is a known problem with the closely related practice of contingent evaluation, which uses willingness-to-pay (WTP) exercises to assign monetary values to non-market resources. (WTP, TTO, and SG all require respondents to value outcomes that are hypothetical). Economist Jerry Hausman has argued that contingent valuation is hopeless, primarily because survey respondents rarely respond out of "stable or well-defined preferences, but are essentially inventing their answers on the fly, in a way which makes the resulting data useless for serious analysis," a remark that could as easily apply to the elicitation of preferences in valuing health [34]. Moreover, a host of biases (e.g., anchoring, range, question-order) have been associated with WTP exercises, biases that would seem equally relevant to TTO and SG methods but have received little attention in the health-state evaluation literature [35]. 
These are all good reasons for skepticism about the value of asking hypothetical patients to evaluate specific health states. However, there is an additional and important set of cases, involving the valuation of congenital or early acquired conditions, where the difficulty of eliciting valid ratings from the public is even greater. To see why, we turn to the concrete case of imagining what it is like to live with the genetic disease phenylketonuria.

\section{Hypothetical Patients and Congenital Disorders: The Case of PKU}

PKU is an autosomal recessive disorder of phenylalanine metabolism. Phenylalanine, an essential amino acid found in all dietary proteins, is necessary for protein synthesis and other biological functions. Because humans do not synthesize it endogenously, they must obtain it from the foods they eat. However, only a small amount of the ingested phenylalanine is necessary for normal growth and development, with the rest ordinarily converted to another amino acid, tyrosine. In PKU, a deficiency of the enzyme phenylalanine hydroxylase results in both a shortage of tyrosine and, more important, an excess of phenylalanine and its metabolites that is toxic to the developing brain. Untreated PKU is associated with profound cognitive impairment and other physical and neuropsychological abnormalities. But in much of the world today, newborns are routinely screened for the condition at birth, with those found to be affected placed from infancy on a special diet.

That diet typically requires about a $95 \%$ reduction in phenylalanine, and hence protein consumption. To imagine being in the situation of an individual with this condition is thus to envisage leading a life where not only meat, fish, eggs, dairy, soy, and nuts should be avoided, but also products containing wheat, rice, and pasta, and where even consumption of most vegetables is limited to very small, measured amounts. In order to obtain enough protein, the person with PKU must also prepare and consume (typically three times daily) a "formula" that contains all the amino acids except phenylalanine plus extra tyrosine. Since free amino acids have poor organoleptic properties, the formula generally tastes and smells bad to the affected individual's parents, friends, and classmates. In most countries, guidelines recommend that this diet be maintained for life.

For the friend or other outsider to whom food is important, that would likely seem a terrible fate-probably a worse state of affairs than it does to the person with PKU. Several years ago, as part of a book project on PKU and newborn screening, I conducted a series of in-depth interviews with patients being followed at the Boston Children's Hospital metabolic clinic. The results were surprising, at least to me-and at that point I had been researching PKU for at least 15 years. Most of the interviewees, as expected, reported great difficulty in adhering to the recommended diet. Indeed, for most adherence was intermittent and partial, as is true for patients with PKU in general [36]. But the reasons for the difficulty were not necessarily the ones I assumed.

Thus, I had thought that the patients would miss such foods as burgers, bacon and eggs, and icecream: the items that I would crave were I for some reason suddenly required to follow their dietary regime. (As Nagel noted of bats, we can only imagine what it would be like for us to navigate through sonar or hang upside down by our feet all day). But since individuals are born with PKU, many have never acquired a taste for such high-protein foods. As adults, they may even find these foods, to which they did not become accustomed in childhood, unpalatable. Indeed, some actually come to like the formula and resent it when the composition is altered to increase its palatability [37].

One major obstacle faced by adolescents and adults in adhering to the PKU diet, especially in countries without national health insurance, is financial. In the US, many states do not mandate 
insurance coverage and, even where some coverage is required, it may be capped and include age and other limitations. Moreover, struggles to obtain reimbursement are common even in those states with broad insurance mandates [38-40]. (For a recent study of the financial and time burden of PKU on affected individuals and their families, see [41]). Yet financial challenges are ignored in even the most sophisticated health-state descriptions [42].

But the major obstacle to adherence, which affects individuals with PKU everywhere, arises from the fact that food is not just about nutrition but is central to our personal and social relationships. Few social occasions do not involve food. Christine Brown, a parent of two sons with PKU, eloquently expresses the isolating effects of dietary treatment. She notes that her boys, who are restricted to six grams of natural protein a day, "cannot eat anything with meat, soy, dairy, nuts, legumes, wheat, or flour," and she asks: "What do you do when you travel to a new country to experience a new culture? You eat their food. What do have to celebrate your birthday? Cake. What did my son's seventh grade football team do to celebrate an undefeated season? A pizza party. How do you celebrate Thanksgiving? Turkey. What do you do on a first date? Go out for dinner." And she goes on to note that "these experiences are not part of my children's world because every single thing that they put into their mouth has to be weighed, measured, and calculated" [43]. Moreover, individuals with PKU must constantly explain to friends, classmates, and co-workers why they can't eat what's on offer. They can never be spontaneous about going out to eat at a restaurant or a friend's house. In general, they feel that they lack control over their lives.

To the extent that struggles with cost, isolation, and lack of autonomy are central features of life with PKU, they could in theory be incorporated into health-state descriptions. But what such descriptions could not even in principle include is a sense of what it is like never to have tasted highprotein foods. Moreover, for the hypothetical patient, the psychological process of rating the quality of life with PKU inevitably involves imagining giving up something that is already possessed. It thus involves a loss, and most cognitive psychologists and behavioral economists believe that humans are strongly loss-averse. In any case, the task of imagining what it is like to live with a congenital or early-acquired condition, including those that are far more disabling such as Duchenne muscular dystrophy, cystic fibrosis, or Krabbe Disease, is of a different order of difficulty from the already difficult task of imagining what it is like to lose a limb or go blind or deaf later in life.

\section{Concluding Remarks}

The 2016 US Panel reiterated its predecessor's conclusion that health-state preferences should generally be obtained "from a representative sample of fully informed members of the community." (The question of what it means to be "fully informed" and whether this criterion is achieved-or achievable-in practice receives remarkably little attention here or elsewhere in the literature [44]). However, the panel diverged from its predecessor in acknowledging several situations where patient-derived preferences should also be gathered, including "cases in which it is difficult for those who have not experienced or observed the health states associated with the condition and/or its treatment to understand sufficiently well to provide meaningful scores for those health states" [9]. It might be argued that, given the cognitive and affective challenges faced by outsiders in general, the ostensible exceptions actually constitute the rule. But even if one believes that the difficulties that beset all efforts to imagine a non-experienced health state could in principle be addressed, for example, through the use of much more elaborated health-state descriptions (which incorporate 
adapted patients' valuations of their health states), more nuanced survey questions, and provision of an opportunity for reflection, the barriers to accurately envisaging life with a congenital or earlyacquired condition are extremely high and possibly insuperable. Moreover, this category is expanding with improved survival in conditions that were once fatal in children.

Thus, there would seem to be a substantial subset of cases where the sole use of communityderived preferences is inappropriate. In general, health economists are resistant to employing patient-derived preferences for reasons that include difficulties in obtaining a representative sample of patients, of achieving consistency and comparability of evaluations, and cost, since if nonpatients are used, ratings can be elicited for multiple health states from the same people simultaneously $[4,12]$. Moreover, apart from such technical and practical factors, patient as well as general-public valuations are subject to systematic biases [11].

The problems with both community and patient ratings have prompted some commentators to propose abandoning preference-based methods of assigning values to health states altogether, substituting direct methods of evaluation. Thus, Paul Dolan and Daniel Kahneman [11] would have economists develop methods for determining how people feel on a moment-to-moment basis (rather than requiring them to predict how they will feel), while Daniel Hausman [32, 33] proposes to avoid all recourse to informant surveys, whether of patients or the general population, and instead involve both experts and deliberative bodies (which would include individuals with experience of the condition) in assigning values to health states based on objective criteria.

Explicating and evaluating these and other specific options for improving the measurement and valuation of health states is well beyond the scope of this paper. However, several policy conclusions can be drawn from the PKU case. First, there is consensus that valuations elicited from randomlychosen members of the public are valid only if survey respondents are "well-informed." But it is exceedingly difficult to construct health-state descriptions that capture the experiences of living with a chronic condition, whether congenital or not, and there is good reason to doubt that the information requirement is commonly met. Second, the difficulty of trying to imagine what it is like to adhere to the PKU diet supports Nagel's claim that the non-affected have, at best, very limited access to the subjective experience of persons with congenital conditions.

Thus, the current practice in CEA of relying on outsiders to assign values to these health states seems clearly inappropriate. The practice of valuing health through the elicitation of preferences is entrenched in health economics and unlikely to be discarded. But that practice would at least be improved were it acknowledged that survey respondents with no personal experience of conditions existing from birth will find it difficult at best "to provide meaningful scores for those health states." Taking the US Panel's own proviso seriously implies that there is an important category where patient-derived preferences should be accorded greater weight in assessing quality of life than is now usually the case.

\section{Acknowledgments}

This project, which had been germinating for some years, came to fruition during a residency at the Brocher Foundation in Hermance, Switzerland: http://www.brocher.ch. The author greatly benefited from conversations with several Brocher colleagues, especially Michal Lavidor of Tel Aviv University and Gert Jan van der Wilt of Radboud University Medical Centre; the latter first suggested the potential relevance of Thomas Nagel's essay, "What is it like to be a bat?" She is also indebted 
to psychologist Susan E. Waisbren and nutritionist Frances Rohr, both formerly of the Boston Children's Hospital Metabolism Program, for helpful comments on the analysis of life with PKU and to Daniel Wikler of the Harvard T.H. Chan School of Public Health for his close reading of a draft of the manuscript and thoughtful criticisms and suggestions.

\section{Author Contributions}

The author did all the research work of this study.

\section{Competing Interests}

The author has declared that no competing interests exist.

\section{References}

1. Nagel T. What is it like to be a bat? Philos Rev. 1974; 83: 435-450.

2. Skotko BG, Levine SP, Macklin EA, Goldstein RD. Family perspectives about Down syndrome. Am J Med Genet A. 2016; 170: 930-941.

3. Emens EF. Framing disability. Univ III Law Rev. 2013; 2012: 1383-1442.

4. Feeny D, Krahn M, Prosser LA, Salomon JA. Online appendix for valuing health outcomes [Internet]. Oxford, NY: Oxford University Press; 2016. Available from: https://healthsci.mcmaster.ca/docs/librariesprovider68/other-publications/07-onlineappendices-valuing-health-outcomes30sep2016(double).pdf?sfvrsn=37c10666 2.

5. Versteegh MM, Brouwer WB. Patient and general public preferences for health states: A call to reconsider current guidelines. Soc Sci Med. 2016; 165: 66-74.

6. Nord E. Cost-value analysis in health care: Making sense out of QALYs. Cambridge: Cambridge University Press; 1999.

7. Nord E, Pinto JL, Richardson J, Menzel P, Ubel P. Incorporating societal concerns for fairness in numerical valuations of health programmes. Health Econ. 1999; 8: 25-39.

8. Helgesson $\mathrm{G}$, Ernstsson $\mathrm{O}$, Åström M, Burström K. Whom should we ask? A systematic literature review of the arguments regarding the most accurate source of information for valuation of health states. Qual Life Res. 2020; 29: 1465-1482.

9. Feeny D, Krahn M, Prosser LA, Salomon A. Valuing health outcomes. In: Cost-Effectiveness in Health and Medicine. 2nd ed. New York: Oxford University Press; 2016. pp.167-199.

10. Gandjour A. Theoretical foundation of patient v. population preferences in calculating QALYs. Med Decis Making. 2010; 30: E57-E63.

11. Dolan P, Kahneman D. Interpretations of utility and their implications for the valuation of health. Econ J. 2008; 118: 215-234.

12. Menzel PT. Utilities for health states: Whom to ask. In: Encyclopedia of Health Economics. San Diego: Elsevier; 2014. pp.417-424.

13. Ogorevc M, Murovec N, Fernandez NB, Rupel VP. Questioning the differences between general public vs. patient based preferences towards EQ-5D-5L defined hypothetical health states. Health Policy. 2019; 123: 166-172.

14. Ubel PA, Loewenstein G, Jepson C. Whose quality of life? A commentary exploring discrepancies between health state evaluations of patients and the general public. Qual Life Res. 2003; 12: 
599-607.

15. Daniels N, Rose S, Zide ED. Disability, adaptation, and inclusion. In: Disability and Disadvantage. New York: Oxford University Press; 2009. pp.54-85.

16. Krabbe PF, Tromp N, Ruers TJ, van Riel PL. Are patients' judgments of health status really different from the general population? Health Qual Life Outcomes. 2001; 9: 31.

17. Peeters $Y$, Stiggelbout AM. Health state valuations of patients and the general public analytically compared: A meta-analytical comparison of patient and population health state utilities. Value Health. 2010; 13: 306-309.

18. Smith DM, Sherriff RL, Damschroder L, Loewenstein G, Ubel PA. Misremembering colostomies? Former patients give lower utility ratings than do current patients. Health Psychol. 2006; 25: 688-695.

19. Albrecht GL, Devlieger PJ. The disability paradox: High quality of life against all odds. Soc Sci Med. 1999; 48: 977-988.

20. Van Gestel YR, Voogd AC, Vingerhoets AJ, Mols F, Nieuwenhuijzen GA, van Driel OR, et al. A comparison of quality of life, disease impact and risk perception in women with invasive breast cancer and ductal carcinoma in situ. Eur J Cancer. 2007; 43: 549-556.

21. Brazier J, Akehurst R, Brennan A, Dolan P, Claxton K, McCabe $C$, et al. Should patients have a greater role in valuing health states? Appl Health Econ Health Policy. 2005; 4: 201-208.

22. Murphy RP, Boyce CJ, Dolan P, Wood AM. Valuing the $Q$ in QALYs: Does providing patients' ratings affect population values? Health Psychol. 2020; 39: 37-45.

23. Schwartz B, Sommers R. Affective forecasting and well-being. In: The Oxford Handbook of Cognitive Psychology. New York: Oxford University Press; 2013. pp.704-716.

24. Flynn E, Hovasapian A, Levine LJ. Affective forecasting. In: Encyclopedia of Health Psychology. New York: Kluwer Academic/Plenum Publishers; 2020. pp.21-29.

25. Lucas RE. Long-term disability is associated with lasting changes in subjective well-being: Evidence from two nationally representative longitudinal studies. J Pers Soc Psychol. 2007; 92: 717-730.

26. Schkade DA, Kahneman D. Does living in California make people happy? A focusing illusion in judgments of life satisfaction. Psychol Sci. 1988; 9: 340-346.

27. McKie J, Hurworth R, Shrimpton B, Richardson J, Bel C. Priority setting and patient adaptation to disability and illness: Outcomes of a qualitative study. Health Care Anal. 2014; 22: 255-271.

28. Ubel PA, Loewenstein G, Jepson C. Disability and sunshine: Can hedonic predictions be improved by drawing attention to focusing illusions or emotional adaptation? J Exp Psychol Appl. 2005; 11: 111-123.

29. Gilbert DT, Wilson TD. Prospection: Experiencing the future. Science. 2007; 317: 1351-1354.

30. Institute for Health Metrics and Evaluation. GBD History [Internet]. Seattle, WA: Institute for Health Metrics and Evaluation; 2019. Available from: http://www.healthdata.org/gbd/about/history.

31. Vos T, Murray C. Measuring the health of populations: The global burden of disease study methods. In: Oxford Textbook of Global Public Health. 6th ed. New York: Oxford University Press; 2015.

32. Hausman DM. Valuing health: A new proposal. Health Econ. 2010; 19: 280-296.

33. Hausman DM. Valuing Health: Well-Being, Freedom, and Suffering. New York: Oxford University Press; 2015. 
34. Hausman J. Contingent valuation: From dubious to hopeless. J Econ Perspect. 2012; 26: 43-56.

35. Ternent L, Tsuchiya A. A note on the expected biases in conventional iterative health state valuation protocols. Med Decis Making. 2013; 33: 544-546.

36. Jurecki ER, Cederbaum S, Kopesky J, Perry K, Rohr F, Sanchez-Valle A, et al. Adherence to clinic recommendations among patients with phenylketonuria in the United States. Mol Genet Metab. 2017; 120: 190-197.

37. Paul DB, Brosco JP. The PKU Paradox: A Short History of a Genetic Disease. Baltimore: Johns Hopkins University Press; 2013.

38. Berry SA, Brown C, Grant M, Greene CL, Jurecki E, Koch J, et al. Newborn screening 50 years later: Access issues faced by adults with PKU. Genet Med. 2013; 15: 591-599.

39. Berry SA, Kenney MK, Harris KB, Singh RH, Cameron CA, Kraszewski JN, et al. Insurance coverage of medical foods for treatment of inherited metabolic disorders. Genet Med. 2013; 15: 978-982.

40. Huntington K, Buist NR. Medical food for treatment of inborn errors of metabolism and state legislative mandates. Top Clin Nutr. 2009; 24: 289-306.

41. Rose AM, Grosse SD, Garcia SP, Bach J, Kleyn M, Simon NJ, et al. The financial and time burden associated with phenylketonuria treatment in the United States. Mol Genet Metab Rep. 2019; 21: 100523.

42. Simon NJ, Richardson J, Ahmad A, Rose A, Wittenberg E, D'Cruz B, et al. Health utilities and parental quality of life effects for three rare conditions tested in newborns. J Patient Rep Outcomes. 2019; 3: 4.

43. Brown CS. Family reflections on phenylketonuria. Pediatr Res. 2018; 84: 797-798.

44. Karimi M, Brazier J, Paisley S. Are preferences over health states informed? Health Qual Life Outcomes. 2017; 15: 105.

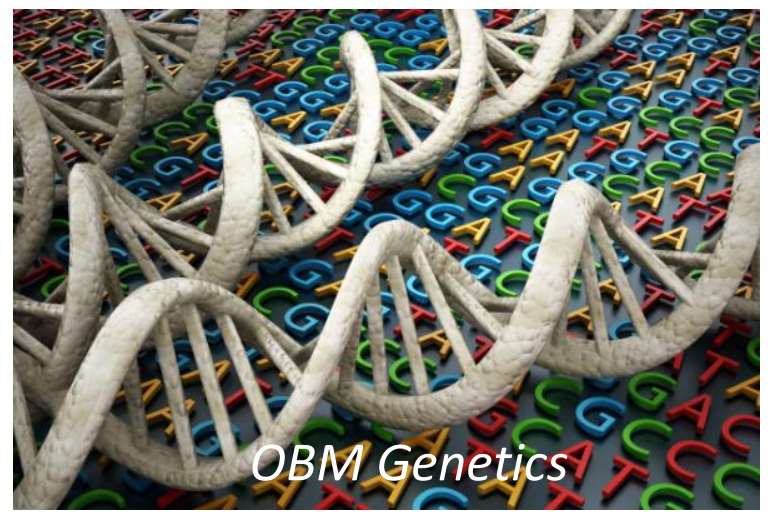

Enjoy OBM Genetics by:

1. Submitting a manuscript

2. Joining in volunteer reviewer bank

3. Joining Editorial Board

4. Guest editing a special issue

For more details, please visit:

http://www.lidsen.com/journals/genetics 